\title{
Measurements Methods for the analysis of Nuclear Reactors Thermal Hydraulic in Water Scaled Facilities
}

\author{
C. Spaccapaniccia, P.Planquart, J.M. Buchlin, von Karman Institute of Fluid Dynamics Belgium
}

\begin{abstract}
The Belgian nuclear research institute (SCK•CEN) is developing MYRRHA. MYRRHA is a flexible fast spectrum research reactor, conceived as an accelerator driven system (ADS). The configuration of the primary loop is pool-type: the primary coolant and all the primary system components (core and heat exchangers) are contained within the reactor vessel, while the secondary fluid is circulating in the heat exchangers. The primary coolant is Lead Bismuth Eutectic (LBE). The recent nuclear accident of Fukushima in 2011 changed the requirements for the design of new reactors, which should include the possibility to remove the residual decay heat through passive primary and secondary systems, i.e. natural convection (NC). After the reactor shut down, in the unlucky event of propeller failures, the primary and secondary loops should be able to remove the decay heat in passive way (Natural Convection). The present study analyses the flow and the temperature distribution in the upper plenum by applying laser imaging techniques in a laboratory scaled water model. A parametric study is proposed to study stratification mitigation strategies by varying the geometry of the buffer tank simulating the upper plenum.
\end{abstract}

Index Terms-Natural Convection Loop, Particle Image Velocimetry, Laser Induced Fluorescence, Pool Type Reactors

\section{INTRODUCTION}

In a Natural Circulation Loop (NCL) the flow is driven by Archimedes' forces and transports heat from a heat source to a heat sink without the aid of mechanical moving parts. Thanks to this, NCLs find applications in many industrial and domestic fields: nuclear reactor core cooling, solar heaters, electronic components cooling, house heater, etc..

In industrial or domestic heat transport systems, the fluid circulates through hydraulic elements for which pressure drops

Paper submission: 1/06/2017

Spaccapaniccia C.*, Planquart P. * and Buchlin J.M*.

*Author for correspondence

Von Karman Institute for Fluid Dynamics,

Rhode Saint Genese, 1640, Belgium, may be relevant: in these fields a thermosyphon loop is most often encountered. A thermosyphon loop is a particular case of NCL in which the heat sink is placed at higher level with respect to the heater's thermal center.

The object of this analysis is the experimental, pool type reactor MYRRHA conceived by the nuclear research institute $\mathrm{SCK} \bullet \mathrm{CEN}$. In the pool type reactor, all the thermo-hydraulic elements of the primary loop are submerged in the primary coolant. Figure 1-a sketches the main elements of the primary loop: 1-core, 2-barrer, 3-upper plenum 4-heat exchanger, 5propellers, 6-lower plenum. The barrel is a perforated cylinder containing the upper core structure (instrumentation and safety bars).

After a shutdown event, the core continues to produces heat by spontaneous decay of radioisotopes. The decay heat is equal to $10 \%$ of the nominal power. In the unlucky event of propellers failure, the decay heat should be removed passively by Natural Convection. Thus, the primary loop of the nuclear reactor MYRRHA operating in passive heat removal conditions can be considered an analogous NCL. The NCL under analysis is a closed, turbulent, single phase thermosyphon where the flow is generated by buoyancy forces. Figure 1 extrapolates and schematizes the NCL starting from a symmetry axis of the pool type reactor. The distance in vertical (y) and horizontal (x) direction between the heating and cooling elements are indicated respectively as $\mathrm{H}$ and $\mathrm{L}$. The sketch considers the presence of the upper plenum acting like a buffer tank interposed between core and heat Exchangers (HE).

Few studies exist on NCL provided with a buffer tank. Furthermore, literature concerning this kind of systems usually involves NCLs with constant diameter where the tank's effect is limited to heat storage (Misra et al. [1]) or temperature and flow stabilization (Naveen et al. [2], Misale et al. [3]). It is therefore important to analyze the flow behavior and temperature distribution inside the buffer tank of the NCL

E-mail: chiara.spaccapaniccia@,vki.ac.be

E-mail: philippe.planquart@vki.ac.be

E-mail: buchlin@vki.ac.be 
outlined in Figure 1-b. In particular, given the buoyancy dominated nature of the flow, thermal stratification is expected to occur in the buffer tank. Stratification and pressure drops in the primary loop are expected to contribute negatively to the NC flow.

The objective of the study was therefore to verify the influence of the geometry of the loop and of the upper vessel on the mass flow. The methodology chosen to address the problem is a parametric study carried out in a 1:8 scaled water model (AQUARIUM) of the of the NCL of Figure 1-b. The study consisted in the simultaneous measurement of mass flow, velocity field and temperature field in different buffer tank configurations, using Thermocouples (TCs) and optical imaging techniques such as PIV (Particles Image Velocimetry) and LIF (Laser Induced Fluorescence).

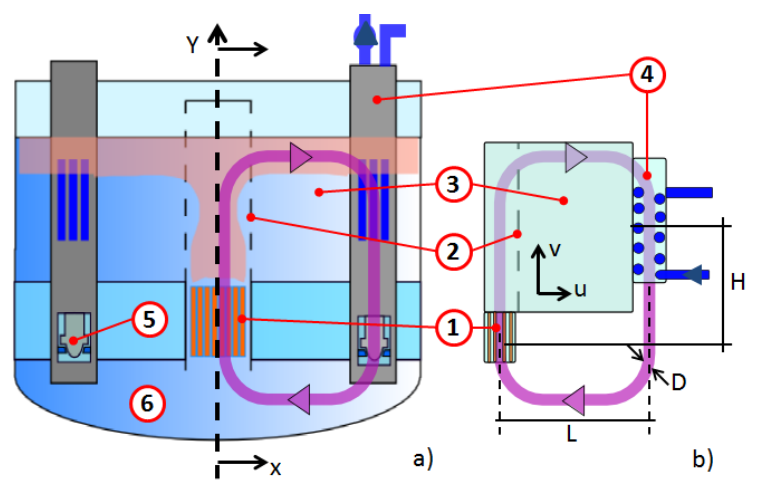

Figure $1 \mathrm{NCL}$ in a pool type reactor

\section{SCALING AND FACILITY DESCRIPTION}

In passive heat removal conditions, a mass flow is established as a consequence of a balance between frictional pressure losses of the hydraulic loop and Archimedes forces induced by heating and cooling power (Welander [4]). The pressure head generated can be defined as: (Todreas[5]):

$$
\Delta P_{B}=\beta \rho\left(T_{h}-T_{c}\right) g H
$$

If the pool geometry is approximated as axial symmetric, the dominating components of velocity are $v$ (vertical) and $u$ (radial) (Figure 1-b). Under this assumption, and with the hypothesis of equal heat provided and removed, the equations of transport of momentum and energy describing the NCL under analysis are:

$$
\begin{gathered}
u \frac{\partial u}{\partial x}+v \frac{\partial u}{\partial y}=-\frac{1}{\rho_{0}} \frac{\partial p_{x}}{\partial x}+v \frac{\partial^{2} u}{\partial x^{2}}+v \frac{\partial^{2} u}{\partial y^{2}} \\
u \frac{\partial v}{\partial x}+v \frac{\partial v}{\partial y}=-\frac{1}{\rho_{0}} \frac{\partial p_{y}}{\partial y}+v \frac{\partial^{2} v}{\partial x^{2}}+v \frac{\partial^{2} v}{\partial y^{2}}-g \beta\left(T_{h}-T_{c}\right)
\end{gathered}
$$

$$
u \frac{\partial T}{\partial x}+v \frac{\partial T}{\partial y}=\alpha \frac{\partial^{2} v}{\partial x^{2}}+\alpha \frac{\partial^{2} v}{\partial y^{2}}
$$

The characteristic lengths chosen for the normalization of $\mathrm{x}$ and $\mathrm{y}$ are $\mathrm{H}$ and $\mathrm{L}$ as indicated in Figure 1-b. $\mathrm{T}_{\mathrm{h}}$ and $\mathrm{T}_{\mathrm{c}}$ are the hottest and coldest temperature achieved in the NCL (usually at core and HE's exit). The characteristic pressure chosen is the overall pressure drop (friction and local losses) in the hydraulic loop $\Delta \mathrm{P}_{\mathrm{ch}}$, therefore:

$$
\begin{aligned}
& U \frac{\partial V}{\partial X}+V \frac{\partial V}{\partial Y}= \\
& -\frac{1}{\rho_{0}} \frac{\Delta P_{c h}}{V_{c h}^{2}} \frac{\partial P_{y}}{\partial Y}+\frac{v}{V_{c h} H}\left(\frac{H^{2}}{L^{2}} \frac{\partial^{2} V}{\partial X^{2}}+\frac{\partial^{2} V}{\partial Y^{2}}\right)-\frac{H g \beta\left(T_{h}-T_{c}\right)}{V_{c h}^{2}} \theta
\end{aligned}
$$

Since in the NCL the buoyant pressure head should balance the pressure drops (Todreas [6]) the expression for the characteristic velocity $\mathrm{V}_{\text {ch }}$ can be determined by balancing the pressure term with the buoyant term of Equation 4:

$$
\begin{gathered}
\frac{H g \beta\left(T_{h}-T_{c}\right)}{V_{c h}^{2}}=-\frac{1}{\rho_{0}} \frac{\Delta P_{c h}}{V_{c h}^{2}}=E u \\
\Rightarrow V_{c h}=\sqrt{\frac{H g \beta\left(T_{h}-T_{c}\right)}{E u}}
\end{gathered}
$$

where $\mathrm{Eu}$ is the Euler number for pressure drops.In the NCL, flow rate, pressure drops and buoyancy forces are not independent quantities, thus it is not possible to know a priory $\mathrm{V}_{\mathrm{ch}}$ and $\Delta \mathrm{P}_{\mathrm{ch}}$. In the rigorous non-dimensional analysis, in order to escape this problem, the characteristic velocity $\mathrm{V}_{\mathrm{ch}}$ (or the characteristic pressure drops $\Delta \mathrm{P}_{\mathrm{ch}}$ ) is chosen in order to obtain $\mathrm{Eu}=1$. For the present case $\mathrm{Eu}=1$ implies $\mathrm{V}_{\mathrm{ch}}=(\operatorname{Hg} \beta \Delta \mathrm{T})^{0.5}$.

Replacing this expression of $\mathrm{V}_{\mathrm{ch}}$ in the normalized equations of momentum and energy we obtain the following nondimensional equations for the steady state:

$$
\begin{gathered}
U \frac{\partial U}{\partial X}+V \frac{\partial U}{\partial Y}=-\frac{\partial P}{\partial X}+\sqrt{\frac{1}{G r_{H}}}\left(A_{R}^{2} \frac{\partial^{2} U}{\partial X^{2}}+\frac{\partial^{2} U}{\partial Y^{2}}\right) \\
U \frac{\partial V}{\partial X}+V \frac{\partial V}{\partial Y}=-\frac{\partial P}{\partial Y}+\sqrt{\frac{1}{G r_{H}}}\left(A_{R}^{2} \frac{\partial^{2} V}{\partial X^{2}}+\frac{\partial^{2} V}{\partial Y^{2}}\right)+\theta \\
U \frac{\partial \theta}{\partial X}+V \frac{\partial \theta}{\partial Y}=\sqrt{\frac{1}{P r \cdot G r_{H}}}\left(A_{R}^{2} \frac{\partial^{2} \theta}{\partial X^{2}}+\frac{\partial^{2} \theta}{\partial Y^{2}}\right)
\end{gathered}
$$

where the appearing dimensionless groups are:

$$
N D_{1}=A_{R}^{2} G r_{H}^{-0.5}, N D_{2}=A_{R}^{2}\left(P r \cdot G r_{H}\right)^{-0.5}
$$

Respecting the similarity of both the groups ND1 and ND2 is possible only if the Pr number of the fluids is the same. However, this is not feasible when studying LBE flow with water facilities. 
The geometry of a nuclear reactor's primary loop is very complex, especially the geometry of the reactor's core, and it is unfeasible to reproduce every geometrical detail with a scaled model. However, the geometry of the upper plenum can be reproduced accurately. The exact matching of the Grashof number is also unfeasible with a low power water model. However, $\mathrm{Gr}_{\mathrm{H}}$ can be relaxed until the flow is in the correct regime (turbulent natural convection for $\mathrm{Gr}_{\mathrm{H}}>10^{9}$ ).

The resulting model (called AquariumII) and its main dimensions are shown in Figure 2. AquariumII is a 1:8, 2D slab model of the MYRRHA's NCL. The facility is approximately 1 $\mathrm{m}$ high and $0.8 \mathrm{~m}$ wide. The flow circulates in the anticlockwise direction. The main elements of the system are:

- Heater: series of flat electrical resistances (maximum total power $=3 \mathrm{~kW}$ ).

- Barrel Plate: an interchangeable perforated plate with circular holes.

- Buffer Plate: a plate with adjustable height to simulate the Heat Exchangers entrance level.

- Heat Exchanger: a copper spiral in which water cooled by an external refrigerating system is circulating.

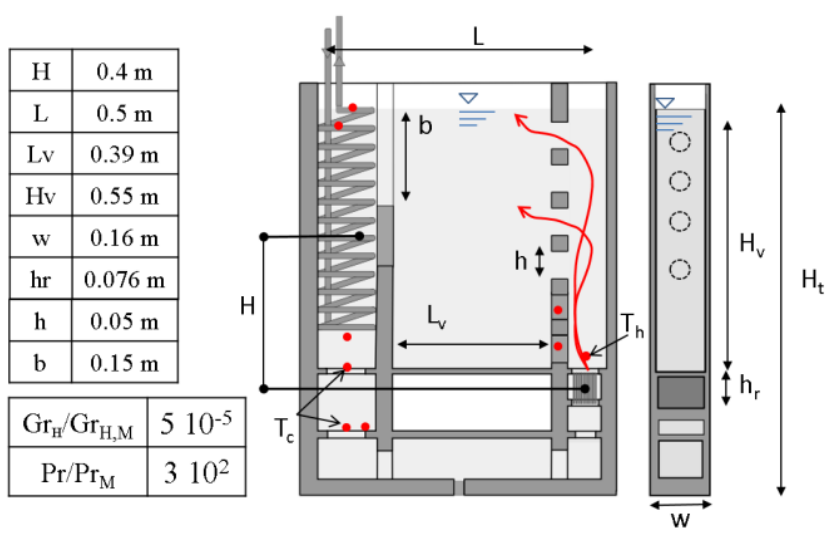

Figure 2 sketch of AQUARIUM II

The peculiarity of the model is that the position or the characteristics of each one of these elements can be changed to carry out a parametric study. The cold leg is accessible through a front window, in order to insert pressure drops elements, in case of necessity. The buffer tank is provided with four optical accesses (top, bottom, front and side) composed of glass windows.

\section{Configurations Tested}

Figure 3 shows the configurations chosen to carry out the parametric study. Configuration 1 was obtained by increasing the free area of the barrel plate through opening all the passages available. Configuration 2 was obtained by shiftiong the heat exchanger entrance towards the bottom of $15 \mathrm{~cm}$. Configuration
3 was obtained by reducing the area above the heater through the immersion of a triangular full shape.
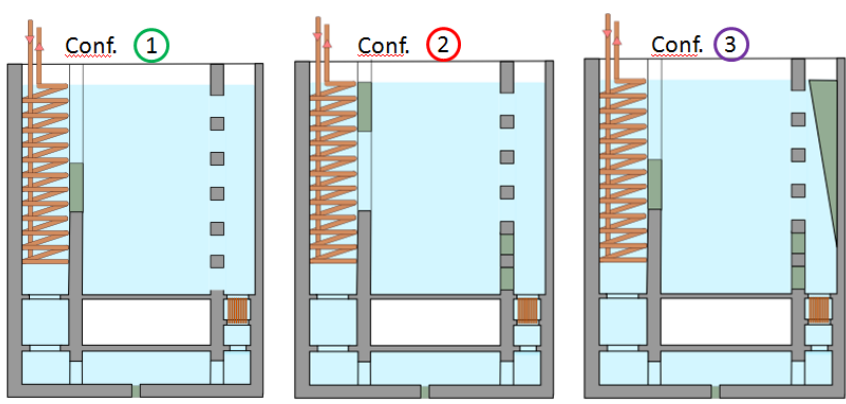

Figure 3 Parametric study configurations

\section{EXPERIMENTAL SET UP AND METHODOLOGY}

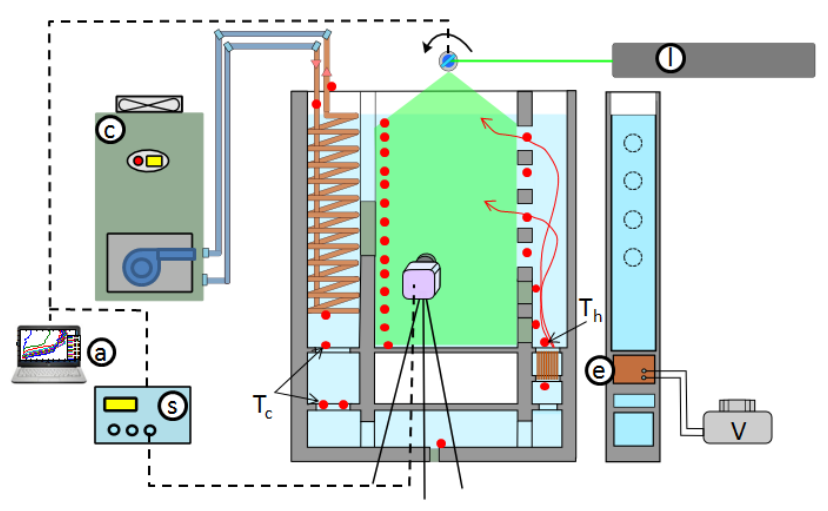

Figure 4 Experimental set up

Figure 4 shows the reference configuration of Aquarium II and the instrumentation arrangement. The TCs are indicated with red dots. Water is seeded with a proper concentration of Rhodamine $\mathrm{B}$ and with fluorescent tracing particles, for temperature and velocity measurements. An Argon-Ion Laser produces a continuous beam picked at $515 \mathrm{~nm}$. The beam power selected is $2.1 \mathrm{~W}$, and it is kept constant during the measurements by the laser remote control. Since it is necessary to measure the full tank test section $(55 \times 39 \mathrm{~cm})$, a rotating mirror was used to scan the test section with the continuous laser beam. The laser beam scans a section of the plenum, while the rolling shutter CCD camera, provided with a high pass filter of $550 \mathrm{~nm}$, detects the fluorescent signal for temperature measurements and the fluorescent light emitted by the particles. Each image acquisition occurs when the disk rotating together with the mirror completes a revolution: an opening in the disk activates a sensor that sends a signal to the synchronizer, which activates the camera, triggering the recording of one exposure. The image acquisition frequency is limited by the rotating mirror speed $(41 \mathrm{~Hz})$. The signal triggering the acquisition of the first image also activates the TCs acquisition, which records temperature values simultaneously to the image acquisition but with an independent and higher frequency $(70 \mathrm{~Hz})$.

The Rhodamine B concentration used for the LIF experiments is $1.6 \mathrm{mg} /$ liter. The PIV tracers seeding the water are fluorescent polyethylene microspheres of the size of $40 \mu \mathrm{m}$ with spectrum 
emission similar to the one of Rhodamine B: in this way a single image will contain information on both temperature (RhB signal) and velocity (particles signal). The concentration of particles is $0.025 \mathrm{~g} /$ liter.

Figure 5 shows the acquisition time line of the simultaneous temperature and velocity measurements performed. Before the beginning of the test, a reference temperature is recorded by means of TCs and a set of reference images is recorded by the CCD camera. The TCs measurement covers all the duration of the experiments and it is divided in 5 different recordings. Every image set includes 2400 frames, covering approximately one minute of experiment. In all the tests performed the heat exchanger and the electrical resistances are switched on simultaneously at one minute after the beginning of the experiment.

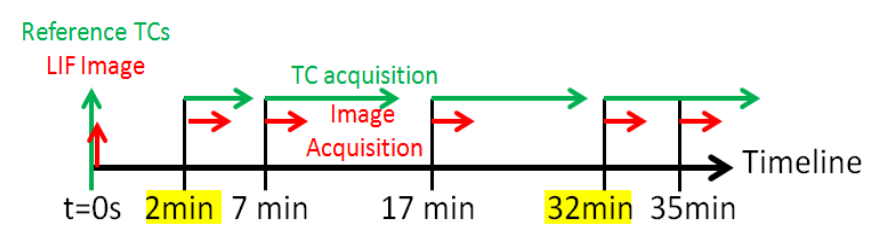

Figure 5 Data acquisition time line.

\section{DATA TREATMENT AND PROCESSING}

One camera imaging techniques require a pre-processing of the images in order to separate the signals of the particles and the signal of the background.

Westerweel et al. [6] and Adrian and Westerweel [7] analysed the histogram of a synthetic PIV image. Westerweel et al. found out the background part of a PIV image can be fit by a normal distribution, while Adrian and Westerweel showed that an exponential approximation can be applied to the particles signal, when the background noise is characterized by lower intensity with respect to the particle signal. The intensity counts of an excited solution of water and $\mathrm{RhB}$ with a low concentration of dye are lower than the intensity counts of a fluorescent particle which contains the same dye, thanks to the light enhancement due to the Mie scattering. Therefore the threshold separating particles and temperature signal can be found on the image histogram, by approximating it with a Gaussian and an exponential distribution (Figure 6).

The approximation is done iteratively using a guess threshold point, until the point of best fitting is found. The result of the procedure is displayed in the images at the top right of Figure 6 , which shows the image before and after the threshold, where the particle signal is successfully removed.

One of the major problems for a LIF image quality is the appearance of striations forming because of gradient of refractive index. Existing literature (Grafsronningenet al. [8], Coolen et al. [9]) suggests to remove geometrical elements like lines by the convolution of the two dimensional Fourier transform of the image with a proper filter. Figure 7 shows the filter used to correct the striations. Once the LIF images are normalized and corrected, a calibration must be used to convert the LIF signal into temperature values. For cases of large facilities like Aquarium II, achieving a uniform temperature in every point of the test section is possible only when the water is at ambient temperature. The calibration is therefore performed by associating the value of normalized intensity measured in small image windows placed nearby the TCs with the temperature measured by the corresponding TC. Figure 8 shows a calibration curved retrieved for five image acquisitions (corresponding to one experiment).

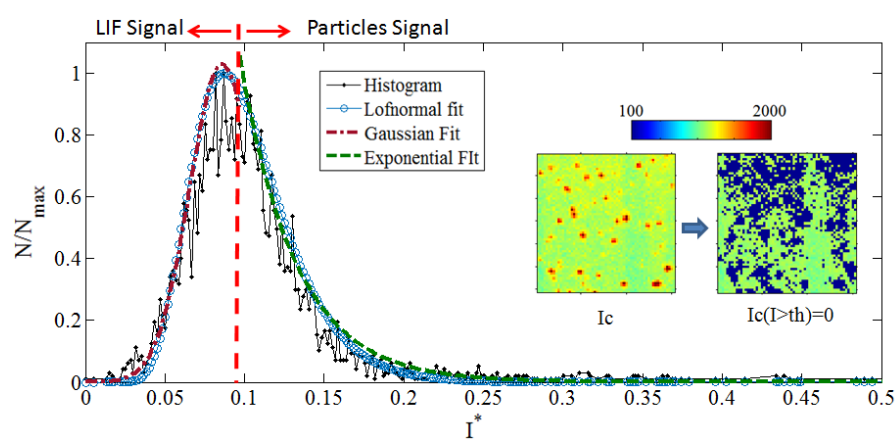

Figure 6 Threshold searching on a PIV-LIF image histogram

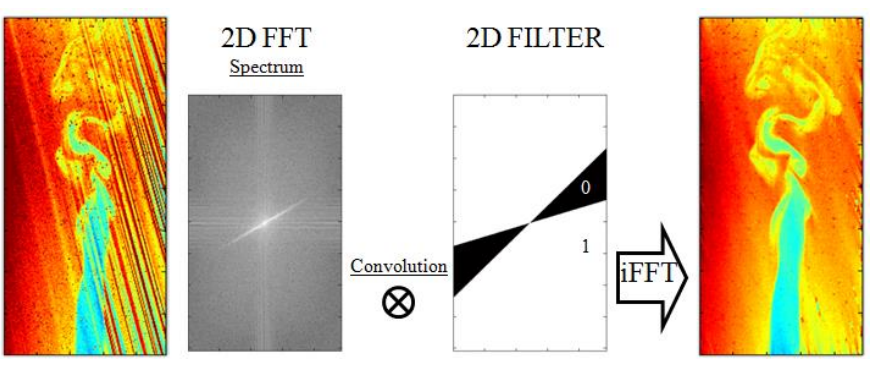

Figure 7: Striations removal logic

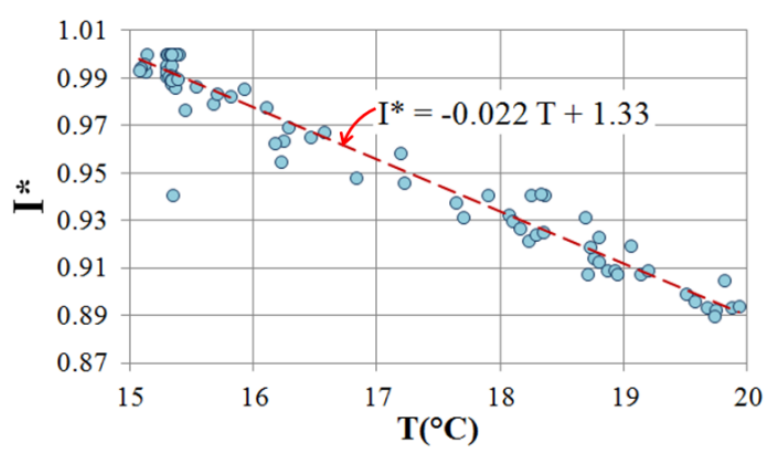

Figure 8 LIF signal-TC signal correlation curve used for calibration

Finally, the parameters of interest allowing to characterize the performance of the loop are loop mass flow and stratification number S. The loop mass flow was calculated through integration of the velocity profiles measured with PIV at every aperture of the barrel.

$$
\dot{m}_{\text {loop }}=2 \pi \rho \sum_{i=1}^{N} \int_{0}^{h / 2} r u(r) d r
$$

The stratification number $\mathrm{S}$ was derived for one line of TCs using the definition of Gandhi et al. [11] (Equation 11). 


$$
S=\frac{1}{J-1} \sum_{j=1}^{J-1} \frac{T_{j+1}-T_{j}}{d y} \frac{(J-1) d y}{T_{\max }-T_{\min }}
$$

\section{RESULTS}

Figure 9 shows the stratification measured in the plenum of Aquarium II. The values of temperature are averaged over one minute of acquisition to match the image acquisition period. The stratification measurements performed with TCs will be discussed together, with the aid of the measured velocity and temperature fields. Considering the group formed by the first six uppermost TCs, the topmost one indicates a lower temperature with respect to the others. This is due to the room conditions: the room temperature was not higher than 15 degrees. The same group of 6 thermocouples shows an increase in temperature along the experiment. The explanation of this can be easily found when observing the streamlines, velocity and temperature fields displayed in Figure 10.

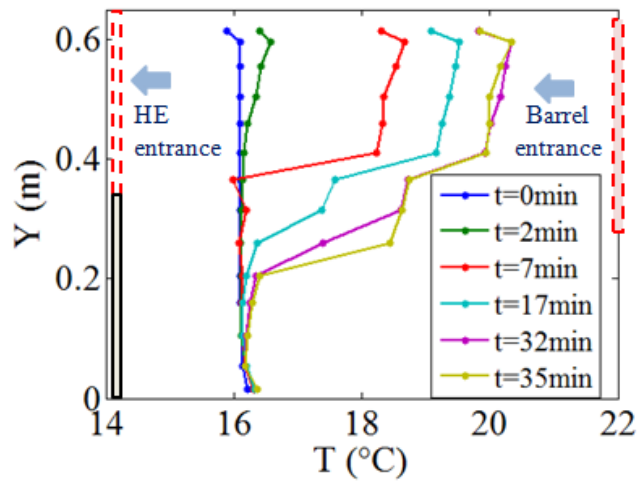

Figure 9 - evolution of temperature profiles measured at the $\mathrm{HE}$ entrance for the reference configuration of Aquarium II

The image on the left of Figure 10 shows the velocity magnitude field measured by laser scanning PIV and the associated streamlines. The image on the right shows the temperature field measured with LIF. The vector field is overlapped to the temperature field.

The six uppermost TCs are immersed in the hot stream coming from the barrel plate. As shown in Figure 10, a strong vortex is produced at the beginning of the test in correspondence of the heat exchanger entrance. At the beginning of the test, the hot fluid coming from the plume region and crossing the barrel plate is highly buoyant since the plenum is not stratified yet, which translates in higher flow momentum. The vortex entrains flow from the heat exchanger region, which is still at initial temperature condition. The result of this is an inclined temperature profile in the first six thermocouple of the plenum. The mass flow re-entering the plenum doesn't possess enough momentum to reach the barrel plate again and it spreads on the lower part of the plenum, opening wider and colliding against the hot flow coming from the bottommost hole of the barrel.

The vortex divides therefore in two flows: one joins the hot buoyant jet outgoing from the barrel, the other deviates toward the bottom of the plenum. The Temperature field of Figure 10 shows lower values of temperature in the lower part of the plenum. This is in accordance with the value of temperature detected by the Thermocouples.
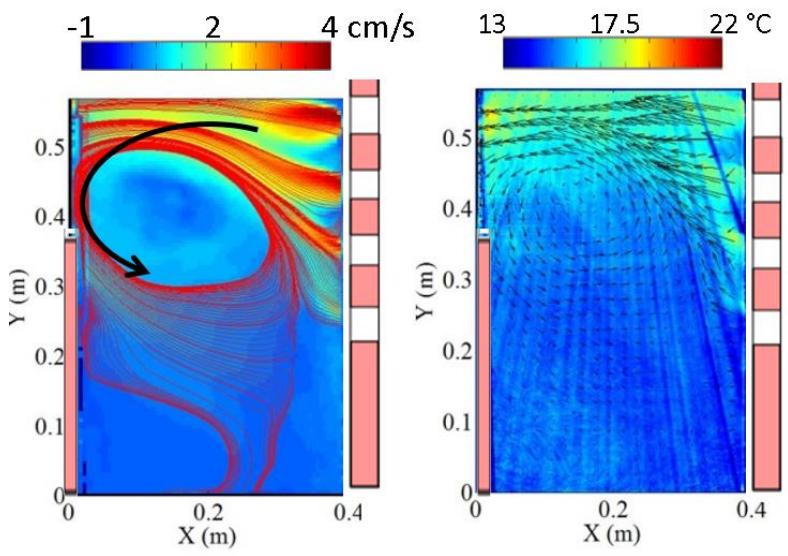

Figure 10 - velocity, streamlines and temperature field at $\mathrm{t}=2 \mathrm{~min}$ for the reference configuration of Aquarium II
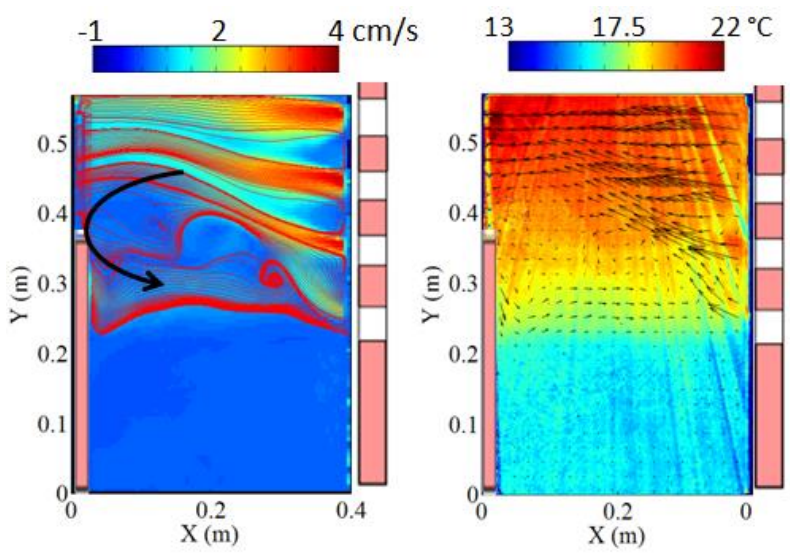

Figure 11 Reference Conf., 32 min after beginning of the test

The temperature field shows that the hot buoyant plumes outgoing from the barrel conquers space on the upper part of the plenum and force the vortex downward. This is consistent with the model of the plume in confined space derived by Baines et al. [10], for which the warm fluid occupies the upper part of the plenum and it is progressively replaced by the incoming fluid, moving towards the bottom.

Figure 11 shows velocity and temperature fields obtained for the reference configuration at 32 minutes after the beginning of the experiment. We can observe horizontal plumes outgoing from the barrel and forming a central vortex: the vortex size is greatly reduced with respect to the beginning of the test. Smaller scale vortices develop at the boundary layers of the lowest horizontal plumes, as a result of Kelvin-Helmotz instability occurring at the shear layer between two streams possessing different values of velocity. A colder mass flow recirculating in the tank form the heat exchanger is detected by both PIV measurements (Figure 11) and TCs measurements (Figure 9). Both The temperature profile measured with the TC array and the LIF data (Figure 11) show very well the separation between the upper mixing region and the bottom, cold and almost motionless region. 
Nevertheless the measurements involved a full transient of the NCL, the velocity and temperature results discussed and compared in the following section are the ones obtained at the end of each experiment.

\section{Effects of increased barrel porosity, Configuration 1}

In Configuration 1 the flow is recalled and entrained in the plume from the bottommost barrel apertures (Figure 12). The hot flow outgoing from the top of the barrel crosses the tank until the bottom, carrying with him the heat of the warm fluid, and re-enter partially the barrel region. The recirculation involves in this case almost all the tank, improving the thermal mixing. and leads to a more uniform temperature profiles (Figure 13, first graph) with respect to the reference configuration.

Configuration 1 shows higher values of loop mass flow with respect to other configurations tested (), indicating better performances. This is due to the communication between the upper heater warm region and the cold bottom tank region, which yields a stack effect: the difference in temperature between the tank fluid and the fluid above the heater causes a difference of pressure which contributes positively to the mass flow.
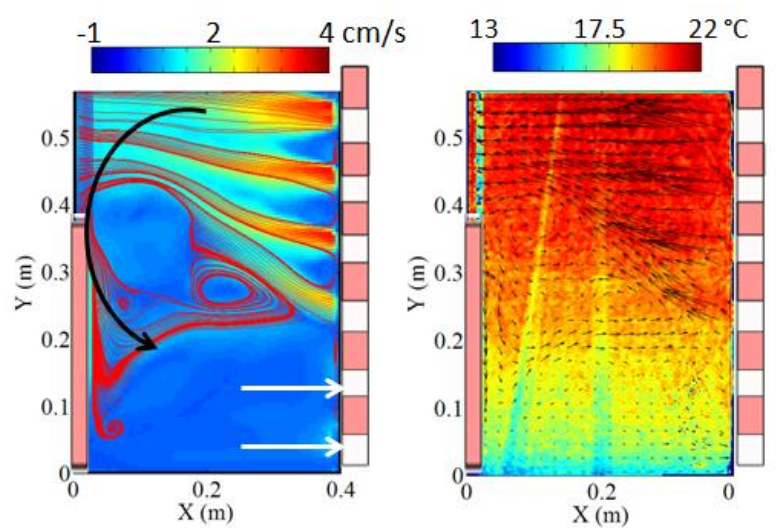

Figure 12 Configuration 1, 32 min after beginning of the test

\section{Effects of HE window position, Conf.2}

The tests performed in the reference configuration and the Conf. 2 show that the position of the central vortex is strongly influenced by the position of the heat exchanger entrance, as can be noticed when comparing the streamlines of Figure 11 and the left plot of Figure 14.

The effects of moving the heat exchanger entrance downwards are mostly negative. For instance, temperatures achieve higher values in the topmost part of the tank (Figure 13-second graph): this is due to the increased blockage effect caused by the presence of an obstacle between the upper tank and the heat exchangers.

On the other hand, the border between motionless region and the mixing region moves towards the bottom increasing the mixing zone (Figure 14-left).
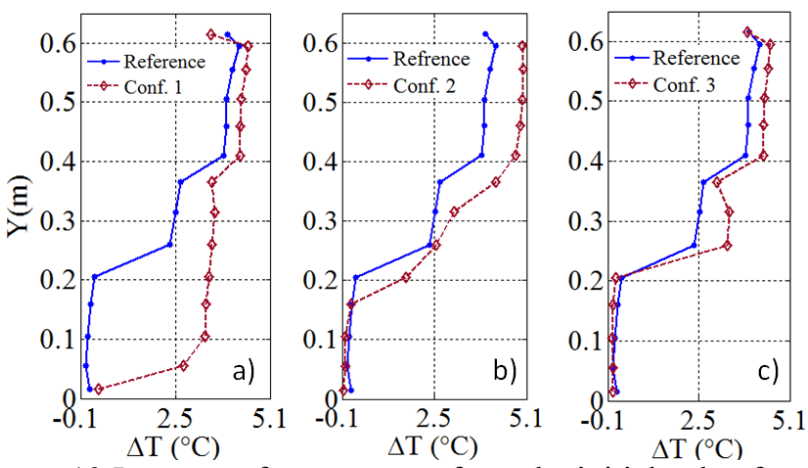

Figure 13 Increase of temperature from the initial value for the different configurations.

Effects of blockage in the barrel, Conf. 3

Figure 14-right shows the velocity magnitude field obtained decreasing progressively the barrel test section by means of a slope-shaped obstacle at 32 minutes after the beginning of the tests. Comparing the field of Figure 14-right with the velocity field of Figure 11 it can be observed that the size of the plume exiting the topmost hole is reduced when the plume passage area in the plume region is reduced, due to the higher resistance encountered by the hot flow in the barrel region.
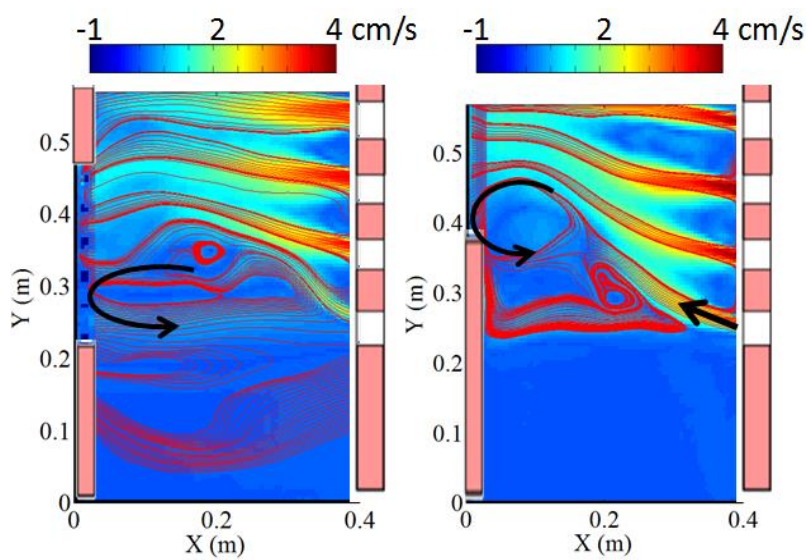

Figure 14 Conf. 2 (left) and Conf. 3 (right) velocity fields, 32 min after beginning of the test
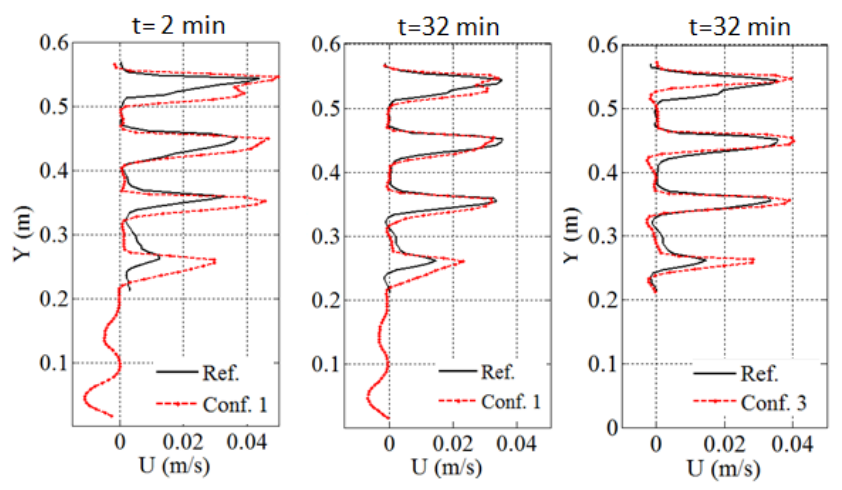

Figure 15 Velocity profiles measured at the barrel exit 
At the same time, the flow outgoing from the bottommost hole becomes more important. In the reference configuration the plume outgoing from the bottommost hole merges immediately after the barrel with the buoyant plumes above, while when the passage area in the barrel is reduced (Configuration 3) this plume in particular assumes a horizontal direction and spread further in the tank.

The last graph of Figure 15 shows the velocity profiles measured at the barrel exit for the Reference geometry and Configuration 3. The effect of redistribution of the mass flow in between the holes is clearly visible in the profiles.

\section{Summary}

The experimental results obtained for the different configurations tested are summarized in Figure 16 in terms of loop mass flow and stratification number. It can be observed that the highest mass flow is achieved for Configuration 1. Configuration 1 is also the only case tested in which the mass flow increases with the time. The lowest mass flow is obtained for Configuration 3, due to the additional flow resistance introduced by decreasing the barrel area. At the end of the transient, Configuration 1 shows the lowest value of stratification number, as expected from the more uniform temperature profiles illustrated in Figure 13.

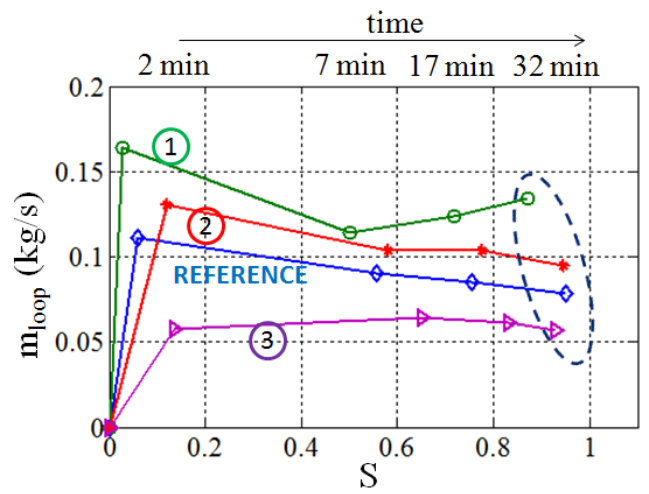

Figure 16 Loop mass flow measured for different configurations along the experiments with $\mathrm{S}$

\section{CONCLUSIONS}

Velocity and temperature measurements with innovative onecamera optical techniques were carried out in the buffer tank of a water NCL simulating passive decay heat removal in a liquid metal pool type reactor. It was observed that the loop mass flow partly completes the loop and partly circulates back in the buffer tank, bypassing the loop. A parametric study was carried out in order to understand the effect of the buffer tank geometry on the stratification. It was observed that the geometry of the plenum effects the temperature gradients and the value of $\mathrm{m}_{\text {loop }}$. The lowest values of $\mathrm{S}$ and the highest values of $\mathrm{m}_{\text {loop }}$ were obtained with Configuration 1. This configuration allows the communication between cold upper plenum and hot barrel duct, yielding to a stack effect. The difference of temperature between these two regions causes a pressure difference which contributes positively to the mass flow. Furthermore, the communication between plenum and barrel duct allows the entrainment of flow in the plume developing right above the heater, reducing the stagnation in the plenum and increasing the mixing. As a result, the plenum temperature profile is more uniform with respect to the reference configuration. As observed thanks to the measurements carried out in Configuration 3 , the progressive reduction of the barrel cross sectional area allows to distribute more uniformly the $\mathrm{m}_{\text {loop }}$ between the barrel apertures without affecting sensibly the temperature distribution in the tank. The flow fields measured in Configuration 2 show that size and position of the convection cell developing at the HE entrance is affected by the heat exchanger window's height and location. The measurements will serve as a base for validating CFD simulations of the NCL. The simulations will allow more extensive parametric studies, leading to the optimization of barrel bottom apertures' size and $\mathrm{HE}$ window position.

\section{NOMENCLATURE}

\begin{tabular}{|c|c|c|}
\hline$x$ & {$[\mathrm{~m}]$} & Cartesian axis direction \\
\hline$y$ & {$[\mathrm{~m}]$} & Cartesian axis direction \\
\hline$r$ & {$[\mathrm{~m}]$} & radius \\
\hline$H$ & {$[\mathrm{~m}]$} & Thermal conductivity \\
\hline$L$ & {$[\mathrm{~m}]$} & Volumetric heat generation density \\
\hline$T_{h}$ & {$[\mathrm{~K}]$} & Interfacial thermal resistance \\
\hline$T_{c}$ & {$[\mathrm{~K}]$} & Temperature \\
\hline$p$ & {$\left[\mathrm{~kg} / \mathrm{ms}^{2}\right]$} & pressure \\
\hline$\Delta P_{c h}$ & {$\left[\mathrm{~kg} / \mathrm{ms}^{2}\right]$} & Characteristic pressure drops \\
\hline$u$ & {$[\mathrm{~m}]$} & Radial velocity \\
\hline$v$ & {$[\mathrm{~m} / \mathrm{s}]$} & Axial velocity \\
\hline$V_{c h}$ & {$[\mathrm{~m} / \mathrm{s}]$} & Characteristic Velocity \\
\hline$\beta$ & {$[1 / \mathrm{K}]$} & Volumetric expansion coefficient \\
\hline$\alpha$ & {$\left[\mathrm{m}^{2} / \mathrm{s}\right]$} & Thermal diffusivity \\
\hline$g$ & {$\left[\mathrm{~m} / \mathrm{s}^{2}\right]$} & Gravity \\
\hline$\rho$ & {$\left[\mathrm{kg} / \mathrm{m}^{3}\right]$} & density \\
\hline$v$ & {$\left[\mathrm{~m}^{2} / \mathrm{s}\right]$} & Kinematic viscosity \\
\hline$m_{\text {loop }}$ & {$[\mathrm{kg} / \mathrm{s}]$} & Loop mass flow \\
\hline$\Theta$ & {$[-]$} & $\mathrm{T}-\mathrm{T}_{\mathrm{c}} / \mathrm{T}_{\mathrm{h}}-\mathrm{T}_{\mathrm{c}}$ \\
\hline \multicolumn{3}{|c|}{ Non DImensional Numbers } \\
\hline$G r_{\mathcal{H}}$ & {$[-]$} & NCL Grashof number \\
\hline $\operatorname{Pr}$ & {$[-]$} & Prandtl number \\
\hline$A_{R}$ & {$[-]$} & Aspect ratio \\
\hline $\mathcal{E} u$ & {$[-]$} & Euler number ofr pressure drops \\
\hline$S$ & {$[-]$} & Stratification number \\
\hline \multicolumn{3}{|c|}{ Abbreviations } \\
\hline$L B E$ & & Eutectic Lead Bismuth \\
\hline$N C L$ & & Natural Convection Loop \\
\hline$P I V$ & & Particle Image Velocimetry \\
\hline$L I F$ & & Laser Induced Fluorescence \\
\hline$T C s$ & & Thermocouples \\
\hline$H E$ & & Heat Hexchangers \\
\hline
\end{tabular}

\section{REFERENCES}

[1] R. Misra. Thermal stratification with thermosyphon effects in solar water heating systems. Energy Conversion and Management, 1994.

[2] K. Naveen, K. N. Iyer, J. B. Doshi, and P. K. Vijayan. Investigations on single-phase natural circulation loop dynamics. Part 2: Role of wall constitutive laws. Progress in Nuclear Energy, 2014

[3] M. Misale, P. Garibaldi, J. C. Passos, and G. Ghisi de Bitencourt. Experiments in a single-phase natural circulation mini-

loop Experimental Thermal and Fluid Science, 2007. 
[4] P. Welander. On the Oscillatory Behaviour of a Differentially Heated Fluid Loop. J. Fluid Mechanic, 1967.

[5] N.E.Todreas, M.S.Kazimi, Nuclear Systems II -Elements of Thermal Hydraulic design

[6] J. Westerweel. On velocity gradients in PIV interrogation. Experiments in Fluids, 2008

[7] R. J. Adrian and J. Westerweel. Particle Image Velocimetry. 2010.

[8] S. Grafsrønningen, A. Jensen. Simultaneous PIV/LIF measurements of a transitional buoyant plume above a horizontal cylinder. International Journal of Heat and Mass Transfer, 2012.

[9] M. C. J. Coolen, R. N. Kieft, C. C. M. Rindt, and A. A. Van Steenhoven. Application of 2-D LIF temperature measurements in water using a Nd: YAG laser. Experiments in Fluids, 1999.

[10] W. Baines and J.S.Turner. Turbulent Natural Convection from a source in a confined region. J. Fluid Mechanic, 1968.

[11] M. Gandhi, J. B. Joshi, and P. K. Vijayan. Study of two phase thermal stratification in cylindrical vessels: $C F D$ simulations and PIV measurements. Chemical Engineering Science, 2013.

[12] C. Spaccapaniccia. Experimental study of natural convective internal flows. PhD thesis, Université Libre de Bruxelles, September 2016. 